\title{
Maternal factors contributing to under-five mortality at birth order 1 to 5 in India: a comprehensive multivariate study
}

\author{
Rajvir Singh ${ }^{1}$ and Vrijesh Tripathi ${ }^{*}$
}

\begin{abstract}
The objective of the study is to assess maternal factors contributing to under-five mortality at birth order 1 to 5 in India. Data for this study was derived from the children's record of the 2007 India National Family Health Survey, which is a nationally representative cross-sectional household survey. Data is segregated according to birth order 1 to 5 to assess mother's occupation, Mother's education, child's gender, Mother's age, place of residence, wealth index, mother's anaemia level, prenatal care, assistance at delivery, antenatal care, place of delivery and other maternal factors contributing to under-five mortality. Out of total 51555 births, analysis is restricted to 16567 children of first birth order, 14409 of second birth order, 8318 of third birth order, 5021 of fourth birth order and 3034 of fifth birth order covering $92 \%$ of the total births taken place 0-59 months prior to survey. Mother's average age in years for birth orders 1 to 5 are 23.7, 25.8, 27.4, 29 and 31 years, respectively. Most mothers whose children died are Hindu, with no formal education, severely anaemic and working in the agricultural sector. In multivariate logistic models, maternal education, wealth index and breastfeeding are protective factors across all birth orders. In birth order model 1 and 2, mother's occupation is a significant risk factor. In birth order models 2 to 5, previous birth interval of lesser than 24 months is a risk factor. Child's gender is a risk factor in birth order 1 and 5 . Information regarding complications in pregnancy and prenatal care act as protective factors in birth order 1 , place of delivery and immunization in birth order 2 , and child size at birth in birth order 4. Prediction models demonstrate high discrimination that indicates that our models fit the data. The study has policy implications such as enhancing the Information, Education and Communication network for mothers, especially at higher birth orders, in order to reduce under-five mortality. The study emphasises the need of developing interventions to address the issues of anaemia, mothers working in the agricultural sector and improving relevant literacy among mothers.
\end{abstract}

Keywords: India; Under-five mortality; Demographic and health survey (DHS); Birth order

\section{Introduction}

Globally, the under-five mortality rates have declined from 85 per 1000 to 51 per 1000 (UNICEF 2012). However, it is estimated that more than 7 million children will die before attaining the age of five. Of these, India, Pakistan, Ethiopia, Nigeria and Democratic Republic of Congo will suffer half of all under-five children deaths (UNICEF 2008). India alone shares the burden of $24 \%$ of world's under-five mortality followed by Nigeria which

\footnotetext{
*Correspondence: vrijesh_tripathi@yahoo.com

${ }^{2}$ Department of Mathematics \& Statistics, The Faculty of Science and Technology, The University of the West Indies, St Augustine Campus, Trinidad \& Tobago, West Indies

Full list of author information is available at the end of the article
}

shares $11 \%$ of this burden (UNICEF 2012). It is obvious that health policies in these five countries need to be reviewed and new impetus provided to bring down the high under-five mortality rate. India has seen a decline in these rates from 124 per 1000 in 1990 to 61 per 1000 in 2011 (UNICEF 2012). However, as part of its commitment to reduce under-five mortality to 41 per 1000 by 2015 (UNICEF 2012), India needs to become a special focus area.

The United Nations Children's Fund (UNICEF) identifies that children are at a greater risk of dying before age five if they are born in rural areas, among the poor, or to a mother deprived of basic education (UNICEF 2012). Even though the National Family Health Survey in India 
(NFHS-3 2007) highlights that the rate of reduction in under-five mortality rates has been higher in rural areas compared to urban areas, these figures are qualified by wealth, educational and gender inequalities (NFHS-3 2007). Moreover, the fact that under-five mortality is not declining at a faster rate points to policy focus areas not yet under coverage. A major cause of concern is the stagnation in reduction of neonatal deaths (Bhaumik 2013; Kumar et al. 2013). Though it has been pointed out that there is need for "investment in new-born care units and intensive care units" (Bhaumik 2013), there is also need to strengthen maternal services for the lengthy prenatal period. This study is conceived to find the socio-demographic factors related to maternal health care. The study aims to identify if the determinants of under-five mortality change according to birth order. ${ }^{\text {a }}$ While birth order plays the role of a strong confounder in under-five mortality, Srinivasan postulated that the intrinsic growth rate and the mean generational length of any population may get affected by the birth order pattern (Srinivasan 1980). For this reason, stratification according to birth order is necessary to properly understand patterns of epidemiological predictors of underfive mortality. Detailed analysis can lead to identification of concerns specific to each birth order since primagravidae are the most at risk. Using birth order as the basis of segregation can have policy implications and reveal specific populations to be targeted for extension of information, education and communication (IEC). Moreover, the World Health Organisation recognises reproductive health as inclusive of "the right of access to appropriate health-care services that will enable women to go safely through pregnancy and childbirth and provide couples with the best chance of having a healthy infant (Glasier et al. 2006)". Hence, it is important to recognise target populations to customise maternal health care in spite of inequalities of rural urban, wealth, gender and education to ensure the health of mother and child with the aim of reducing under-five mortality.

\section{Materials and methods}

\section{Data}

Data file IAKR52FL.SAV on children records was accessed from Demographic and Health Survey (DHS) site with due permission. The sample was weighted for population differences, rural-urban and slum and non-slum proportional differences to arrive at a nationally representative data in NFHS - 3 conducted on the DHS format used across 80 countries (NFHS-3 2007). The nationally representative household based sample was created through stratified, multistage clustering sampling strategy with two stages at rural level and three stages at urban level. Information on children based on mother's self-report of birth and death history of children born are included in the study from records of all interviewed women 15-49 years in 29 states in 6 regions (NFHS -3 2007). A total of 51555 of first to fifteenth birth order occurred in the period. The average birth order of children in the sample was $2.6 \pm 1.8$. Hence, it was decided to restrict the study from birth order 1 to 5 . A final total of 16567 children of first birth order, 14409 of second birth order, 8318 of third birth order, 5021 of fourth birth order and 3034 of fifth birth order capturing $92 \%$ of the total births during 59 months preceding the survey are available for the analysis. In case of multiple births, only first birth was included in the analysis (NFHS -3 2007).

\section{Explanatory variables}

Mother's age at index child in years, gender of child at index birth order (male, female), place of residence (rural, urban), religion (Hindu, Muslim, Christian and others), type of caste (scheduled caste, scheduled tribe, other backward classes and Others), standard of living (low, medium, and high), mother's occupation (non-working, service and agriculture), mother's anaemia level (no anaemia, severe, moderate, and mild), prenatal care (yes, no), assistance at delivery (yes, no), antenatal care (home, govt. hospital, private hospital and village/NGO), information regarding complications in pregnancy (yes, no), pregnancy health nutrition education (yes, no), Breastfeeding health nutrition education (yes, no), place of delivery (home, hospital /others), breastfeeding (yes, no), immunization (yes, no), child's age in months ( $<1$ month, 1-12 months and $>$ 12 months), birth interval ( $<24$ months, 24-36 months and $>36$ months) and region ( North, Central, East, North East, West and South) are all taken as explanatory variables as defined by the NFHS-3. For purposes of univariate and multivariate analysis, a few variables are modified/ merged into lesser categories for better understanding of results. Wealth index (poorest, poorer, middle, richer and richest) is recoded as poorest and poor, middle and richer and richest. Mother's and father's education is coded as no formal education and primary, secondary and greater than higher secondary. Father's occupation (non-working, service and agriculture) is taken as part of the definition of the socio-economic status of the family. Size of child at birth (very large, larger than average, smaller than average and very small) are recoded as larger than average, average and smaller than average. All these variables are taken as independent variables and child's status (live or dead) as dependent variable in the study. Child's age is calculated as difference in months between the date of birth and the date of interview for living child and between the date of birth and the date of death of child as reported by the mother (NFHS-3 2007). 


\section{Statistical analysis}

Frequency tabulations describe the characteristics of under-five children at each birth order in the study. It is observed that most of the deaths in the data occur within one month or at the time of birth. Cox Proportional Hazard assumptions assume a linear relationship exists between the endpoint and predictor variables. Further, recall bias would be present in the data due to the retrospective nature of the data collection. Due to nonfulfillment of assumptions and the possibility of recall bias in the current data set that could cause misleading estimates, logistic regression is chosen as the appropriate statistical tool for analysis. Logistic regression is used to determine factors associated with the mortality forcing child age in months and mother's age at index child into multivariate analysis at each birth order as known confounders. Significant variables at p-value $\leq 0.10$ and modified appropriate categories are considered for multivariate logistic regression analysis after checking co-linearity among the explanatory variables. Forward stepwise logistic regression methods are applied to assess significant explanatory variables of under-five mortality for each birth order. Cross-validation of confidence intervals calculated for odds ratios derived by multivariate logistic regression analysis are internally validated via bootstrapping re-sampling methods using 100re-samples for each birth order model (Kleinbaum et al. 1998). The developed model's ability to discriminate was assessed using Area Under the Receiver Operating Characteristic (AUROC) (Bewick et al. 2005). The AUROC, ranging from 0.5 to 1.0 , justifies the probabilistic model by describing its ability to reliably predict the event, in this case, under-five mortality. P-value $\leq 0.05$ (two tailed) is considered for statistical significant level. SPSS 20.0 statistical package is used for the analysis (SPSS2011).

\section{Results}

Child status (live or dead) is taken as dependent variable and percentage of deaths in parenthesis with distribution of each category of variable for each birth order are provided in Table 1. Mother's average age for birth order 1 to 5 are 23.7 years, 25.8 years, 27.4 years, 29 years and 31 years, respectively. Most mothers whose children died are Hindu, with no formal education, severely anaemic and working in the agricultural sector. A regional distribution of under-five mortality shows that it is higher for birth order 1 than 2 in most regions. However, under-five mortality is higher in Central and East regions than in North, North East, West and South regions for all birth orders.

Unadjusted odds ratios and 95\% CI of Univariate analysis are presented in Table 2. In Birth order 1, living in rural area increases the risk to under-five mortality by $73 \%$ (OR, 1.73; 95\% CI, 1.50 - 2.00) compared to urban living areas. Increased wealth index and standard of living increases the chance of under-five survival significantly. Compared with no formal education, the higher the level of mother's education and father's education, the greater is the chance of under-five survival. Mother's occupation in agriculture increases the risk of under-five mortality by $80 \%$ (OR, 1.79; $95 \%$ CI, 1.52 - 2.10). Female child has $22 \%$ (OR, 0.78; $95 \%$ CI, 0.68 - 0.89), Christians have 34\% (OR, 0.66; 95\% CI, 0.50 - 0.87) and all other castes have a better chance of surviving than males, Hindus and scheduled castes, respectively. Breastfeeding (OR, 0.42; 95\% CI, $0.37-0.48)$ and immunization (OR, 0.76; 95\% CI, $0.60-0.98$ ) are protective factors. Breastfeeding health nutrition education increases survival by $24 \%$ (OR, $0.76 ; 95 \%$ CI $0.40-1.43$ ). Children of non-anaemic mothers have a 38\% (OR, 0.62; 95\% CI, 0.37 1.02) better chance of survival than mothers with severe anaemia. Hospital delivery, prenatal care and information regarding complications in pregnancy increase the chance of under-five survival compared to home delivery, no care, and no information regarding complications in pregnancy respectively. A below average size of child at birth significantly increases the chance of mortality than an average or above average size of child at birth. Antenatal care increases the chance of survival than no care. Private hospitals perform better than governmental facilities.

In Birth Order 2, mother's education and occupation, mother's anaemia, place of residence, caste, breastfeeding, immunization, wealth index, father's education, standard of living, place of delivery, size of child at birth, prenatal care and previous birth interval greater than 2 years are significant factors. In Birth Order 3, mother's education, religion, mother's anaemia, place of residence, caste, breastfeeding, wealth index, father's education, standard of living, place of delivery, size of child at birth and birth interval are significant factors.

In Birth order 4, mother's education, religion, gender, breastfeeding, wealth index, standard of living, size of child at birth and birth interval are statistically significant. Breastfeeding, wealth index and standard of living increase the chance of under-five survival. A below average size of child at birth increases the risk of dying by two and a half times compared to an above average size of child at birth (OR, 2.56, 95\% CI, 1.80-3.65). A birth interval of greater than 24 months increases under-five survival significantly. Muslim religion acts as a protective factor in under-five survival compared to Hindu religion. Female children are at a 26\% (OR, 1.26; 95\% CI, 1.01 - 1.58) greater risk of dying than male children.

In Birth Order 5, religion, mother's anaemia, gender, breastfeeding, size of child at birth and birth interval are statistically significant. The most notable absences at birth order 5 are mother's and father's education. Muslims have a 33\% (OR, 0.67; 95\% CI, 0.46- 0.98) better chance of survival than Hindus. Others have a risk factor at $14 \%$ 
Table 1 Distribution of covariates across birth order (1-5) of children and their percentage of deaths in parenthesis

\begin{tabular}{|c|c|c|c|c|c|c|}
\hline \multirow[t]{2}{*}{ Variables } & \multirow{2}{*}{$\begin{array}{l}\text { Category } \\
\text { Total }\end{array}$} & \multicolumn{5}{|c|}{ Birth order } \\
\hline & & $1(16567)$ & 2 (14409) & $3(8318)$ & $4(5021)$ & $5(3034)$ \\
\hline Mother's age at index child & Continuous & $23.7 \pm 4.2$ & $25.8 \pm 4.3$ & $27.4 \pm 4.3$ & $29 \pm 4.3$ & $31 \pm 4.5$ \\
\hline \multirow[t]{2}{*}{ Gender of child } & Male & $8474(6.3)$ & $7521(4.4)$ & $4447(5)$ & $2621(5.6)$ & 1556(5.8) \\
\hline & Female & 8093(5) & 6888(4.6) & $3871(5.1)$ & $2400(7.0)$ & 1478(8.3) \\
\hline \multirow[t]{2}{*}{ Place of residence } & Rural & $9282(6.9)$ & $8366(5.2)$ & $5429(5.4)$ & $3484(6.5)$ & $2246(6.9)$ \\
\hline & Urban & $7285(4.1)$ & $6043(3.5)$ & $2889(4.3)$ & 1537(5.8) & $788(7.2)$ \\
\hline \multirow[t]{4}{*}{ Religion } & Hindu & $12062(5.8)$ & $10409(4.7)$ & $5678(5.1)$ & $3213(6.9)$ & 1876(7.7) \\
\hline & Muslim & $2231(6)$ & 2062(3.9) & $1450(5.8)$ & $1026(4.1)$ & $660(5.3)$ \\
\hline & Christian & 1431(3.9) & 1238(3.9) & $827(3.6)$ & $585(6.2)$ & $377(6.1)$ \\
\hline & Others & $830(5.2)$ & $684(3.5)$ & $358(4.5)$ & $191(6.8)$ & 115(8.7) \\
\hline \multirow[t]{4}{*}{ Type of caste } & Scheduled caste & $2759(6.8)$ & 2543(5.9) & 1591(5.9) & $962(7.3)$ & 594(7.9) \\
\hline & Scheduled tribe & $2204(6.7)$ & 1924(5.2) & 1437(5.0) & 1074(7.1) & $705(6.5)$ \\
\hline & Other backward classes & $5298(6.0)$ & $4714(4.4)$ & 2794(4.8) & $1644(6.0)$ & $1038(7.2)$ \\
\hline & Others & $5634(4.4)$ & $4676(3.5)$ & $2140(4.5)$ & $1122(4.9)$ & $586(6.8)$ \\
\hline \multirow[t]{5}{*}{ Wealth index } & Poorest & 1890(10.8) & 1872(7.2) & 1597(6.2) & 1303(8.1) & $969(7.8)$ \\
\hline & Poorer & 2412(8.7) & $2287(7.0)$ & 1713(7.2) & 1194(5.9) & $776(5.4)$ \\
\hline & Middle & $3231(6.5)$ & 2945(4.4) & 1854(4.4) & $1115(4.9)$ & $649(8.3)$ \\
\hline & Richer & $4048(4.1)$ & $3569(3.8)$ & 1859(3.8) & $930(6.9)$ & $444(4.1)$ \\
\hline & Richest & $4986(2.9)$ & $3736(2.2)$ & $1295(3.5)$ & 479(4.0) & 196(7.7) \\
\hline \multirow[t]{3}{*}{ Standard of living } & Low & 2554(9.8) & $2677(6.9)$ & $2184(6.7)$ & $1745(7.4)$ & $1232(8.4)$ \\
\hline & Medium & $4531(6.4)$ & $4302(5.1)$ & 2888(4.6) & 1814(6.1) & $1122(6.6)$ \\
\hline & High & 7372(3.7) & $6077(2.5)$ & 2642(3.9) & 1152(5.4) & $566(5.8)$ \\
\hline \multirow[t]{4}{*}{ Mother's education } & No formal education & $4122(9.4)$ & $4468(7.4)$ & $3879(6.1)$ & 3099(6.8) & $2143(7.4)$ \\
\hline & Primary & $2242(7.4)$ & $2160(5.1)$ & 1438(5.6) & $742(5.7)$ & $405(6.2)$ \\
\hline & Secondary & $8058(4.2)$ & $6374(2.9)$ & $2721(3.5)$ & 1112(5.1) & $471(6.2)$ \\
\hline & Higher & 2144(2.1) & $1407(1.2)$ & $280(2.5)$ & $68(4.4)$ & $15(6.7)$ \\
\hline \multirow[t]{4}{*}{ Father's education } & No formal education & 2409(9.7) & 2598(6.9) & $2180(5.7)$ & 1734(6.8) & $1286(7.4)$ \\
\hline & Primary & 2016(7.9) & 1967(6.2) & 1362(5.9) & $892(6.7)$ & $531(7.7)$ \\
\hline & Secondary & $8944(4.9)$ & $7532(4.0)$ & $4037(4.7)$ & $2091(5.9)$ & 1073(6.5) \\
\hline & Higher & $3010(3.0)$ & 2171(1.9) & $645(3.1)$ & 247(3.6) & $100(4.0)$ \\
\hline \multirow[t]{3}{*}{ Mother's occupation } & Non-working & 12080(5.1) & 9836(3.8) & $5059(4.8)$ & $2770(5.8)$ & $1531(6.5)$ \\
\hline & Service & 2079(5.2) & 1995(5.1) & 1192(5.6) & $770(7.0)$ & 472(8.9) \\
\hline & Agriculture & 2400(8.8) & $2564(6.5)$ & 2057(5.4) & $1480(6.8)$ & $1031(6.9)$ \\
\hline \multirow[t]{3}{*}{ Father's occupation } & Non-working & 243(6.6) & $133(2.3)$ & $75(5.3)$ & $45(4.4)$ & 18(11.1) \\
\hline & Service & $12626(5.1)$ & 10908(4.2) & $5881(5.1)$ & $3336(6.2)$ & 1904(7.1) \\
\hline & Agriculture & $3610(7.5)$ & $3315(5.5)$ & $2332(4.7)$ & $1625(6.4)$ & $1100(6.8)$ \\
\hline \multirow[t]{4}{*}{ Mother's anemia level } & Severe & 218(7.8) & $211(10.9)$ & 134(11.2) & $98(10.2)$ & $70(12.9)$ \\
\hline & Moderate & $2415(7.7)$ & 2119(6.6) & $1222(6.5)$ & $770(8.3)$ & 518(9.7) \\
\hline & Mild & $5801(5.9)$ & $5050(4.4)$ & $3045(5.2)$ & 1873(5.8) & 1094(5.9) \\
\hline & Not anaemic & 6603(5.0) & $5729(3.8)$ & $3085(4.1)$ & 1727(5.6) & $1000(6.7)$ \\
\hline \multirow[t]{2}{*}{ Prenatal care } & No & $945(5.8)$ & 1194(5.1) & $1271(3.5)$ & 1040(4.6) & $828(3.9)$ \\
\hline & Yes & $944(3)$ & $4676(3.5)$ & $2140(4.5)$ & $1122(4.9)$ & $586(6.8)$ \\
\hline \multirow[t]{2}{*}{ Antenatal care } & Home & $757(4.5)$ & $880(2.3)$ & $538(3.5)$ & $308(2.6)$ & 178(5.1) \\
\hline & Govt. hospital & $3876(3.4)$ & $4432(2.8)$ & 2598(3) & 1499(3.6) & $780(5.4)$ \\
\hline
\end{tabular}


Table 1 Distribution of covariates across birth order (1-5) of children and their percentage of deaths in parenthesis (Continued)

\begin{tabular}{|c|c|c|c|c|c|c|}
\hline & Private hospital & $4606(2.4)$ & 4219(1.9) & $1752(2.2)$ & $858(4.2)$ & $433(3.5)$ \\
\hline & Village/NGO & 188(3.7) & 188(2.7) & $126(0.8)$ & $53(1.9)$ & 33(3.0) \\
\hline \multirow[t]{2}{*}{ Information regarding complications in pregnancy } & No & $6294(3.6)$ & $6735(2.4)$ & $3746(2.8)$ & 2103(3.9) & 1193(4.9) \\
\hline & Yes & $3148(1.9)$ & 2993(2.2) & $1276(2.4)$ & 619(3.1) & 233(3.9) \\
\hline \multirow[t]{2}{*}{ Pregnancy health Nutrition education } & No & $1574(4.1)$ & 1371(3.6) & 915(3.6) & $534(5.1)$ & $352(5.4)$ \\
\hline & Yes & 1474(4.6) & 1368(3.4) & $765(3.3)$ & $415(3.6)$ & $211(4.7)$ \\
\hline \multirow[t]{2}{*}{ Breastfeeding health nutrition education } & No & 1300(1.9) & $1131(2.2)$ & $764(2.1)$ & $450(2.4)$ & $279(4.3)$ \\
\hline & Yes & $1090(1.5)$ & 1049(2.4) & $582(2.2)$ & $309(2.3)$ & $163(5.5)$ \\
\hline \multirow[t]{5}{*}{ Size of child at birth } & Very large & $592(6.6)$ & $577(3.8)$ & $308(4.9)$ & $191(6.8)$ & 109(2.8) \\
\hline & Larger than average & $3181(4.8)$ & 2848(3.9) & $1519(4.1)$ & $880(3.9)$ & $527(6.3)$ \\
\hline & Average & $9040(4.5)$ & $8008(3.6)$ & $4725(4.4)$ & 2853(5.3) & $1689(6.7)$ \\
\hline & Smaller than average & $2425(6.2)$ & 1932(5.4) & $1140(6.1)$ & $705(5.3)$ & $442(7.5)$ \\
\hline & Very small & 1056(10.9) & $801(11.4)$ & $467(10.7)$ & 295(15.3) & $211(13.3)$ \\
\hline \multirow[t]{2}{*}{ Place of delivery } & At home & $6115(7.9)$ & 7019(5.7) & $5406(5.5)$ & $3716(6.1)$ & $2423(6.8)$ \\
\hline & At Hospital/others & 10418(4.1) & $7372(3.2)$ & $2906(4.2)$ & $1300(6.2)$ & $607(7.6)$ \\
\hline \multirow[t]{2}{*}{ Assistance at delivery } & No & $35(25.7)$ & $58(3.4)$ & $64(3.1)$ & $53(9.4)$ & $53(0)$ \\
\hline & Yes & $16495(5.4)$ & $14327(4.4)$ & $8247(5)$ & $4961(6.1)$ & 2976(7.1) \\
\hline \multirow[t]{2}{*}{ Breastfeeding } & No & $5457(9)$ & $5474(6.5)$ & $2942(8.0)$ & $1762(10.2)$ & 1034(12.3) \\
\hline & Yes & $11110(4.0)$ & $8935(3.2)$ & $5376(3.4)$ & $3259(4.1)$ & $2000(4.3)$ \\
\hline \multirow[t]{2}{*}{ Immunization } & No & $7333(5.3)$ & $6099(4.4)$ & $3260(5.1)$ & $1785(6.7)$ & $1029(6.7)$ \\
\hline & Yes & 1813(4.1) & $1296(2.5)$ & 595(4.0) & $287(6.3)$ & $153(5.2)$ \\
\hline \multirow[t]{3}{*}{ Child age in months } & $<1$ month & $3844(16.9)$ & $3127(12.8)$ & $1725(12.7)$ & 1055(15.6) & $627(17.4)$ \\
\hline & 1- 12 months & $3390(6.7)$ & 2993(6.3) & $1720(8.5)$ & $912(11.6)$ & $609(11.7)$ \\
\hline & $>12$ months & $9333(0.6)$ & $8289(0.7)$ & $4873(1.1)$ & $3054(1.4)$ & $1798(1.8)$ \\
\hline \multirow[t]{3}{*}{ Birth interval } & $<24$ months & - & $4204(6.5)$ & 2197(7.9) & $1291(10.1)$ & 756(11.8) \\
\hline & 24-36 months & - & $4707(4.0)$ & $2964(4.5)$ & $1833(5.7)$ & $1150(6.9)$ \\
\hline & $>36$ months & - & $5380(3.1)$ & 3152(3.6) & 1896(4.1) & $1128(4.0)$ \\
\hline \multirow[t]{6}{*}{ Region } & North & $3009(4.8)$ & $2717(4.3)$ & $1589(5)$ & $856(6.5)$ & $487(6)$ \\
\hline & Central & 2969(8.9) & $2744(6)$ & 1978(7.4) & $1428(7.4)$ & 989(9.8) \\
\hline & East & $2566(7.3)$ & 2083(5.2) & $1301(5.4)$ & $854(5.6)$ & $541(5.9)$ \\
\hline & North East & $2965(4.5)$ & 2422(4.6) & $1573(4.7)$ & $1083(6.5)$ & $628(5.6)$ \\
\hline & West & 2191(3.8) & 1825(3.6) & $832(4.2)$ & $389(5.7)$ & $214(4.7)$ \\
\hline & South & $2867(4.3)$ & 2618(3) & 1045(3.8) & $411(3.2)$ & $175(5.7)$ \\
\hline
\end{tabular}

(OR, 1.14; 95\% CI, 0.58- 2.22) compared to Hindus but is not statistically significant. Mother's anaemia effects under-five survival inversely. A female child is at $48 \%$ (OR, 1.48; 95\% CI, 1.12- 1.96) greater risk of mortality than a male child. Breastfeeding increases the chance of under-five survival. A below average size of child at birth increases the risk by 2 times (OR, 2.00; 95\% CI, 1.29 - 3.14) compared with above average size of child at birth. A birth interval greater than 24 months increases under-five survival compared to lesser birth interval.
Most fathers whose children died are poor, with low standard of living, no formal education and working in agricultural sector for birth order 1 and 2 . However, fathers either working or in agriculture had similar effects for birth order 3 to 5 . There are very few fathers who are non-working and this did not show any effect on under-five mortality.

Adjusted odds ratios with $95 \%$ CI for multivariate analysis are provided in Table 3 for each birth order model. Multivariate model for birth order 1 has female, higher mother's education, Christian and richer and richest wealth index as significant protective factors. Breastfeeding (OR, 
Table 2 Univariate analysis of child mortality across birth order (1-5)

\begin{tabular}{|c|c|c|c|c|c|c|}
\hline Variables & Categories & $\begin{array}{l}\text { Birth order-1 } \\
\text { Unadjusted } \\
\text { OR \& Cl 95\% }\end{array}$ & $\begin{array}{l}\text { Birth order -2 } \\
\text { Unadjusted } \\
\text { OR \& Cl } 95 \%\end{array}$ & $\begin{array}{l}\text { Birth order -3 } \\
\text { Unadjusted } \\
\text { OR \& Cl 95\% }\end{array}$ & $\begin{array}{l}\text { Birth order -4 } \\
\text { Unadjusted } \\
\text { OR \& Cl } 95 \%\end{array}$ & $\begin{array}{l}\text { Birth order }-5 \\
\text { Unadjusted } \\
\text { OR \& Cl } 95 \%\end{array}$ \\
\hline Mother's age & Continuous & $0.93(0.91-0.94)$ & $0.93(0.91-0.95)$ & $0.95(0.93-0.98)$ & $0.97(0.95-0.99)$ & $0.99(0.96-1.02)$ \\
\hline Gender of child & Female & $0.78(0.68-0.89)$ & $1.04(0.89-1.21)$ & $1.03(0.85-1.26)$ & $1.26(1.01-1.58)$ & $1.48(1.12-1.96)$ \\
\hline Place of residence & Rural & $1.73(1.50-2.00)$ & $1.50(1.27-1.78)$ & $1.27(1.02-1.57)$ & $1.12(0.87-1.45)$ & $0.96(0.69-1.31)$ \\
\hline \multirow[t]{3}{*}{ Religion } & Muslim & $1.03(0.85-1.25)$ & $0.81(0.64-1.03)$ & $1.15(0.89-1.47)$ & $0.57(0.41-0.80)$ & $0.67(0.46-0.98)$ \\
\hline & Christian & $0.66(0.50-0.87)$ & $0.81(0.60-1.10)$ & $0.81(0.48-1.03)$ & $0.88(0.61-1.27)$ & $0.78(0.49-1.22)$ \\
\hline & Others & $0.88(0.65-1.22)$ & $0.73(0.48-1.11)$ & $0.73(0.52-1.46)$ & $0.98(0.55-1.75)$ & $1.14(0.58-2.22)$ \\
\hline \multirow[t]{3}{*}{ Caste } & Scheduled tribe & $0.97(0.78-1.22)$ & $0.87(0.67-1.12)$ & $0.84(0.61-1.15)$ & $0.97(0.70-1.36)$ & $0.81(0.53-1.24)$ \\
\hline & $\begin{array}{l}\text { Other backward } \\
\text { classes }\end{array}$ & $0.87(0.72-1.05)$ & $0.74(0.59-0.91)$ & $0.80(0.61-1.04)$ & $0.82(0.60-1.12)$ & $0.91(0.62-1.33)$ \\
\hline & Others & $0.62(0.51-0.76)$ & $0.58(0.46-0.73)$ & $0.75(0.56-1.10)$ & $0.66(0.46-0.95)$ & $0.85(0.55-1.32)$ \\
\hline \multirow[t]{2}{*}{ Wealth index } & Middle & $0.65(0.55-0.77)$ & $0.61(0.49-0.75)$ & $0.64(0.49-0.83)$ & $0.68(0.50-0.93)$ & $1.17(0.84-1.63)$ \\
\hline & Richer \& richest & $0.33(0.28-0.39)$ & $0.40(0.34-0.48)$ & $0.53(0.42-0.67)$ & $0.63(0.63-1.08)$ & $0.70(0.47-1.04)$ \\
\hline \multirow[t]{2}{*}{ Standard of living } & Medium & $0.63(0.52-0.75)$ & $0.72(0.60-0.88)$ & $0.67(0.53-0.86)$ & $0.80(0.62-1.04)$ & $0.77(0.57-1.06)$ \\
\hline & High & $0.36(0.30-0.43)$ & $0.35(0.28-0.43)$ & $0.57(0.44-0.74)$ & $0.71(0.52-0.97)$ & $0.68(0.45-1.02)$ \\
\hline \multirow[t]{3}{*}{ Mother's education } & Primary & $0.76(0.63-0.92)$ & $0.67(0.54-0.84)$ & $0.90(0.70-1.17)$ & $0.82(0.58-1.15)$ & $0.83(0.53-1.28)$ \\
\hline & Secondary & $0.42(0.36-0.49)$ & $0.38(0.31-0.45)$ & $0.55(0.40-0.69)$ & $0.74(0.55-0.99)$ & $0.82(0.55-1.24)$ \\
\hline & Higher & $0.21(0.15-0.28)$ & $0.15(0.09-0.25)$ & $0.39(0.10-0.84)$ & $0.63(0.20-2.01)$ & $0.89(0.12-6.87)$ \\
\hline \multirow[t]{3}{*}{ Father's education } & Primary & $0.80(0.65-0.98)$ & $0.89(0.70-1.13)$ & $1.04(0.78-1.39)$ & $0.99(0.72-1.36)$ & $1.05(0.71-1.54)$ \\
\hline & Secondary & $0.48(0.41-0.57)$ & $0.56(0.46-0.68)$ & $0.82(0.65-1.03)$ & $0.86(0.66-1.11)$ & $0.88(0.64-1.21)$ \\
\hline & Higher & $0.29(0.22-0.37)$ & $0.27(0.20-0.38)$ & $0.53(0.33-0.85)$ & $0.52(0.26-0.03)$ & $0.52(0.18-1.45)$ \\
\hline \multirow[t]{2}{*}{ Mother's occupation } & Service & $1.02(0.83-1.26)$ & $1.35(1.08-1.69)$ & $1.19(0.90-1.57)$ & $1.23(0.90-1.70)$ & $1.40(0.96-2.04)$ \\
\hline & Agriculture & $1.79(1.52-2.10)$ & $1.75(1.45-2.11)$ & $1.14(0.91-1.44)$ & $1.18(0.91-1.53)$ & $1.06(0.77-1.45)$ \\
\hline \multirow[t]{2}{*}{ Father's occupation } & Service & $0.76(0.46-1.28)$ & $1.90(0.60-6.00)$ & $0.94(0.34-2.65)$ & $1.43(0.34-5.94)$ & $0.65(0.14-2.70)$ \\
\hline & Agriculture & $1.15(0.68-1.93)$ & $2.53(0.80-8.00)$ & $0.88(0.32-2.45)$ & $1.47(0.35-6.15)$ & $0.59(0.13-2.60)$ \\
\hline \multirow[t]{3}{*}{ Mother's anaemia } & Moderate & $0.99(0.50-1.66)$ & $0.57(0.36-0.91)$ & $0.56(0.31-0.99)$ & $0.80(0.40-1.61)$ & $0.72(0.34-1.55)$ \\
\hline & Mild- anaemia & $0.74(0.45-1.23)$ & $0.38(0.24-0.59)$ & $0.43(0.25-0.76)$ & $0.54(0.27-1.07)$ & $0.42(0.20-0.89)$ \\
\hline & No- anaemia & $0.62(0.37-1.02)$ & $0.32(0.21-0.51)$ & $0.34(0.19-0.60)$ & $0.52(0.26-1.04)$ & $0.49(0.23-1.02)$ \\
\hline Prenatal care & Yes & $0.50(0.37-0.68)$ & $0.45(0.34-0.60)$ & $0.78(0.55-1.10)$ & $0.79(0.55-1.12)$ & $1.23(0.80-1.88)$ \\
\hline \multirow[t]{3}{*}{ Antenatal care } & Govt. hospital & $0.75(0.51-1.10)$ & $1.20(0.78-2.03)$ & $0.83(0.50-1.36)$ & $1.40(0.66-2.98)$ & $1.07(0.51-2.24)$ \\
\hline & Private hospital & $0.53(0.36-0.78)$ & $0.83(0.51-1.36)$ & $0.62(0.36-1.09)$ & $1.64(0.76-3.57)$ & $0.67(0.29-1.57)$ \\
\hline & Village/NGO & $0.82(0.36-1.88)$ & $1.18(0.44-3.17)$ & $0.21(0.03-1.65)$ & $0.72(0.09-5.89)$ & $0.59(0.07-4.79)$ \\
\hline $\begin{array}{l}\text { Information regarding } \\
\text { complications in pregnancy }\end{array}$ & Yes & $0.52(0.39-0.70)$ & $0.90(0.67-1.20)$ & $0.83(0.53-1.25)$ & $0.80(0.48-1.31)$ & $0.79(0.38-1.61)$ \\
\hline $\begin{array}{l}\text { Pregnancy health } \\
\text { nutrition education }\end{array}$ & Yes & $1.12(0.80-1.60)$ & $0.94(0.63-1.41)$ & $0.90(0.53-1.53)$ & $0.70(0.37-1.34)$ & $0.87(0.40-1.91)$ \\
\hline $\begin{array}{l}\text { Breastfeeding health } \\
\text { nutrition education }\end{array}$ & Yes & $0.76(0.40-1.43)$ & $1.08(0.62-1.89)$ & $1.07(0.51-2.34)$ & $0.93(0.36-2.41)$ & $1.30(0.54-3.16)$ \\
\hline \multirow[t]{2}{*}{ Size of child at birth } & Average & $0.88(0.74-1.05)$ & $0.92(0.75-1.14)$ & $1.02(0.78-1.33)$ & $1.23(0.88-1.71)$ & $1.40(0.94-2.12)$ \\
\hline & Below average & $1.54(1.27-1.86)$ & $1.93(1.54-2.42)$ & $1.80(1.34-2.40)$ & $2.56(1.80-3.65)$ & $2.00(1.29-3.14)$ \\
\hline Place of delivery & Hospital & $0.50(0.44-0.57)$ & $0.54(0.46-0.64)$ & $0.75(0.61-0.94)$ & $1.02(0.78-1.32)$ & $1.13(0.80-1.59)$ \\
\hline Breastfeeding & Yes & $0.42(0.37-0.48)$ & $0.48(0.41-0.56)$ & $0.40(0.33-0.49)$ & $0.38(0.30-0.48)$ & $0.32(0.24-0.43)$ \\
\hline Immunization & Yes & $0.76(0.60-0.98)$ & $0.57(0.40-0.82)$ & $0.78(0.51-1.21)$ & $0.92(0.56-1.55)$ & $0.77(0.36-1.63)$ \\
\hline
\end{tabular}


Table 2 Univariate analysis of child mortality across birth order (1-5) (Continued)

\begin{tabular}{lllllll}
\hline Child age in months & $1-12$ months & $0.35(0.30-0.41)$ & $0.60(0.50-0.73)$ & $0.64(0.52-0.80)$ & $0.70(0.55-0.92)$ & $0.63(0.45-0.87)$ \\
& $>12$ month & $0.03(0.20-0.04)$ & $0.46(0.38-0.56)$ & $0.08(0.05-0.10)$ & $0.07(0.05-0.11)$ & $0.09(0.06-0.13)$ \\
Birth interval & $24-36$ months & - & $0.60(0.50-0.73)$ & $0.54(0.41-0.70)$ & $0.53(0.41-0.70)$ & $0.55(0.40-0.76)$ \\
& $>36$ months & - & $0.46(0.38-0.56)$ & $0.43(0.34-0.55)$ & $0.38(0.28-0.51)$ & $0.31(0.22-0.45)$ \\
\hline
\end{tabular}

Reference Category: Gender of Child: Male; Place of residence: Rural; Religion: Hindu; Caste: Schedule Caste; Wealth Index: Poorer \& poorest; Standard of living: Low; Women education: No formal education; Father Education: No formal Education; Mother Occupation: Non-Working; Father Occupation: Non-Working; Mother Smoking: No; Mother Anemia: Severe; Prenatal Care: No; Antenatal Care: Home; Information regarding complications in pregnancy: No; Pregnancy Health Nutrition Education: No; and Breastfeeding Health Nutrition: No. Size of Child at birth: Above average; Place of delivery: Home; Breastfeeding: No; Immunization: No; Pregnancy Complication: No and Birth Interval: $<24$ months. ; Child age in months: $<1$ month and Birth Interval: $<24$ months.

0.04, 95\% CI, 0.03-0.05) and prenatal care (OR, 0.04, 95\% CI, 0.03-0.06) improve under-five survival by $96 \%$. Secondary mother's education, Muslim, middle wealth index and information regarding complications in pregnancy are also protective factors. Mother's occupation in service (OR, 1.25, 95\% CI, 0.92-1.70) or agriculture (OR, 1.35, 95\% CI, 1.051.73 ) is a risk factor. Multivariate model for birth order 2 has place of delivery, primary and above mother's education, richer and richest wealth index, immunization and previous birth interval greater than 24 months as highly significant factors in under-five survival. Mother's occupation in service (OR, 1.89, 95\% CI, 1.43-2.50) or agriculture (OR, $1.51,95 \%$ CI, 1.20-1.93) are risk factors. Multivariate model for birth order 3 has secondary mother's education, middle and richer and richest wealth index, breastfeeding and previous birth interval greater than 24 months as highly significant factors. Multivariate model for birth order 4 has secondary mother's education, Muslim religion, breastfeeding and previous birth interval greater than 24 months as highly significant factors. Below average child size at birth is a significant risk factor (OR, 2.20, 95\% CI, 1.423.34). Multivariate model for birth order 5 has richer and richest wealth index, breastfeeding and previous birth interval greater than 24 months as protective significant factors for under-five survival. Female gender of child is a significant risk factor (OR, 1.61, 95\% CI, 1.16-2.23).

Bootstrapping analysis showed negligible bias in estimated parameters. The ROC curves for different models are shown in Figure 1. The AUROC was 0.94 (95\% CI, 0.93-0.96), 0.89(0.87 - 0.90), 0.86 (0.84 - 0.88), 0.85 (0.82 $0.88)$ and $0.84(0.81-0.87)$ for the developed models 1 to 5 , respectively.

\section{Discussion}

Increasing maternal age is a protective factor in under-five survival. Average maternal age is significantly high for under-five survival in birth order 1 to 5 . This is a good sign in a country that has been fraught with issues of child marriages and early and/ or unwanted pregnancies. Almost half of 20-24 year old women in India (44.5\%) are married before age 18 , and $22 \%$ of all 20-24 year old women have given birth by age 18 years (NFHS-3 2007). However, our study shows a mean age at birth order 1 to be 23.7 years at the national level which means that awareness has improved later pregnancies in many other populations.

The effects of breastfeeding and birth interval greater than 24 months are significant protective factors from birth order 2 to 5 . This is in line with the general perception and is a well- documented fact (Robert et al. 2003; WHO 2000; Gareth et al. 2003; Singh et al. 2012a; Singh et al. 2012b). The UNICEF report projects that if all birth to pregnancy intervals were 3 years, approximately 1.6 million under-five deaths could be prevented annually (UNICEF 2012). Efforts should continue to delay the next pregnancy.

Mother's education is a protective factor for birth order 1 to 4 . However, it did not emerge as a significant factor in birth order 5 because socio-cultural factors other than education dominate the couple's choice of having children. This may be explained by the fact that most families decide their fertility choices early on and hence education plays a relatively small role at higher birth order for under-five survival. However, maternal education plays an important part in utilization of antenatal care (Kumar et al. 2013; Chalasani 2012; Mazumdar 2010; Pradhan et al. 2010; Gakidou et al. 2010; Boone et al. 2006).

Mothers' working in agriculture sector is a risk factor in birth order 1 and 2 in univariate and multivariate analysis. Working mothers are also at risk in birth order 2. However, there was no significant risk in birth order 3 to 5 . Women should be advised against heavy labour especially in the rural agricultural sector. Headey et al. (2012) noted that agricultural workers have the lowest BMIs compared with non-agricultural workers, even after controlling for wealth, health, education, and location. Another study from Kenya by Mustafa and Odimegwu (2008) reports a higher rate of under-five mortality for mothers working in agriculture compared to those not working. Our study specifically identifies mothers at birth order 1 and 2 working in agriculture as being at risk. Further studies are needed to see the reasons for the poor survival of children born to mothers working in the agricultural sector.

The univariate analysis reveals that under-five survival is significantly better in Others (that is, higher castes) compared with scheduled castes. However, it is not a statistically significant factor in multivariate analysis for 
Table 3 Multivariate analysis of child mortality across birth order (1-5)

\begin{tabular}{|c|c|c|c|c|c|c|}
\hline Variables & Categories & $\begin{array}{l}\text { Birth order -1 } \\
\text { Adjusted } \\
\text { OR \& Cl 95\% }\end{array}$ & $\begin{array}{l}\text { Birth order -2 } \\
\text { Adjusted } \\
\text { OR \& Cl 95\% }\end{array}$ & $\begin{array}{l}\text { Birth order }-3 \\
\text { Adjusted } \\
\text { OR \& Cl 95\% }\end{array}$ & $\begin{array}{l}\text { Birth order -4 } \\
\text { Adjusted } \\
\text { OR \& CI 95\% }\end{array}$ & $\begin{array}{l}\text { Birth order }-5 \\
\text { Adjusted } \\
\text { OR \& Cl } 95 \%\end{array}$ \\
\hline Gender of child & Female & $0.76(0.63-0.91)$ & & & & $1.61(1.16-2.23)$ \\
\hline \multirow[t]{3}{*}{ Religion } & Muslim & $0.84(0.63-1.12)$ & & & $0.53(0.35-0.88)$ & \\
\hline & Christian & $0.44(0.30-0.64)$ & & & $0.70(0.44-1.10)$ & \\
\hline & Others & $0.70(0.45-1.08)$ & & & $1.00(0.49-2.00)$ & \\
\hline \multirow[t]{2}{*}{ Wealth index } & Middle & $0.87(0.67-1.12)$ & $0.80(0.61-1.03)$ & $0.56(0.41-0.77)$ & $0.61(0.42-0.90)$ & $0.91(0.61-1.34)$ \\
\hline & Richer \& richest & $0.61(0.47-0.79)$ & $0.59(0.45-0.77)$ & $0.43(0.31-0.60)$ & $0.42(0.86-1.24)$ & $0.45(0.28-0.71)$ \\
\hline \multirow[t]{3}{*}{ Mother's education } & Primary & $1.07(0.82-1.41)$ & $0.66(0.50-0.87)$ & $0.90(0.66-1.24)$ & $0.57(0.37-0.88)$ & \\
\hline & Secondary & $0.79(0.62-1.01)$ & $0.37(0.29-0.48)$ & $0.53(0.38-0.73)$ & $0.53(0.35-0.79)$ & \\
\hline & Higher & $0.31(0.20-0.50)$ & $0.09(0.05-0.16)$ & $0.35(0.15-0.83)$ & $0.20(0.04-0.95)$ & \\
\hline \multirow[t]{2}{*}{ Mother's occupation } & Service & $1.25(0.92-1.70)$ & $1.89(1.43-2.50)$ & & & \\
\hline & Agriculture & $1.35(1.05-1.73)$ & $1.51(1.20-1.93)$ & & & \\
\hline Prenatal care & Yes & $0.04(0.03-0.06)$ & & & & \\
\hline $\begin{array}{l}\text { Information regarding } \\
\text { complications in pregnancy }\end{array}$ & Yes & $0.66(0.47-0.92)$ & & & & \\
\hline Size of child at birth & Average & & & & $1.37(0.92-2.03)$ & \\
\hline \multirow[t]{2}{*}{ Place of delivery } & Hospital & & $0.72(0.58-0.91)$ & & & \\
\hline & Below average & & & & $2.20(1.42-3.34)$ & \\
\hline Breastfeeding & Yes & $0.04(0.03-0.05)$ & $0.08(0.06-0.10)$ & $0.08(0.06-0.11)$ & $0.07(0.44-0.90)$ & $0.08(0.05-0.11)$ \\
\hline Immunization & Yes & & $0.44(0.30-0.65)$ & & & \\
\hline \multirow[t]{2}{*}{ Birth interval } & 24-36 months & - & $0.56(0.45-0.70)$ & $0.45(0.34-0.60)$ & $0.50(0.37-0.70)$ & $0.50(0.34-0.73)$ \\
\hline & $>36$ months & - & $0.35(0.28-0.45)$ & $0.30(0.22-0.42)$ & $0.25(0.17-0.36)$ & $0.21(0.14-0.35)$ \\
\hline
\end{tabular}

Reference Category: Gender of Index Child: Male; Religion: Hindu; Wealth Index: Poorer \& poorest; Mother's education: No formal education; Mother Occupation: Non-Working; Prenatal Care: No; Information regarding complications in pregnancy: No; Place of delivery: Home; Breastfeeding: No; Immunization: No and Birth Interval: $<24$ months.

Note: Mother's age and child's age in months are forced into multivariate analysis at each birth order.

any birth order. Religion acted differentially in different birth orders. Christians have a better chance at survival in birth order 1 and 3 while Muslims survive better at birth order 4 and 5, compared with Hindus. Multivariate analysis supported univariate analysis with Christians and Muslims having better survival than Hindus in birth order 1 and 4, respectively. This agrees with other studies who found that Hindus were disadvantaged in securing the survival of their babies at early ages compared to Muslims and women belonging to other religious groups (Kumar et al. 2013; Bhalotra et al. 2009).

Mothers having severe anaemia are a risk factor to under-five survival across all birth orders in univariate analysis. This reflects the need of early interventions to improve anaemia levels and nutrition index to achieve better results. However, it is not a significant factor in multivariate analysis. Below average size of child is a significant factor in multivariate analysis for birth order 4 . The finding is supported by Kumar and colleagues but they did not analyse the factor by birth order (Kumar et al. 2013).
Female children have a better chance of survival than male children in birth order 1 . Gender has no visible distinction in birth order 2 and 3, but female children are considerably at risk of dying in birth order 4 and 5 . The sex of the previous child or children is unfavourable for the female child (Sawyer 2012; Arokiasamy 2004). This sustains the idea that couples often desire at least one male child and the risk of female child dying at birth order 4 and 5 is very high. In multivariate analysis, female child has a better chance of survival in birth order 1 while it is a risk factor in birth order 5 . This is a matter of grave concern since other researchers (Jha et al. 2006) have also noted the increasing discrepancies in sex ratios in both urban and rural areas. Another study reported that all-cause mortality rate in children aged 1-59 months was about 36\% higher in girls than in boys and most of the leading causes of death were between $12 \%$ and $72 \%$ higher in girls than in boys, with the exception of injuries and meningitis/ encephalitis (Bassani et al. 2010). These facts show the need to identify 

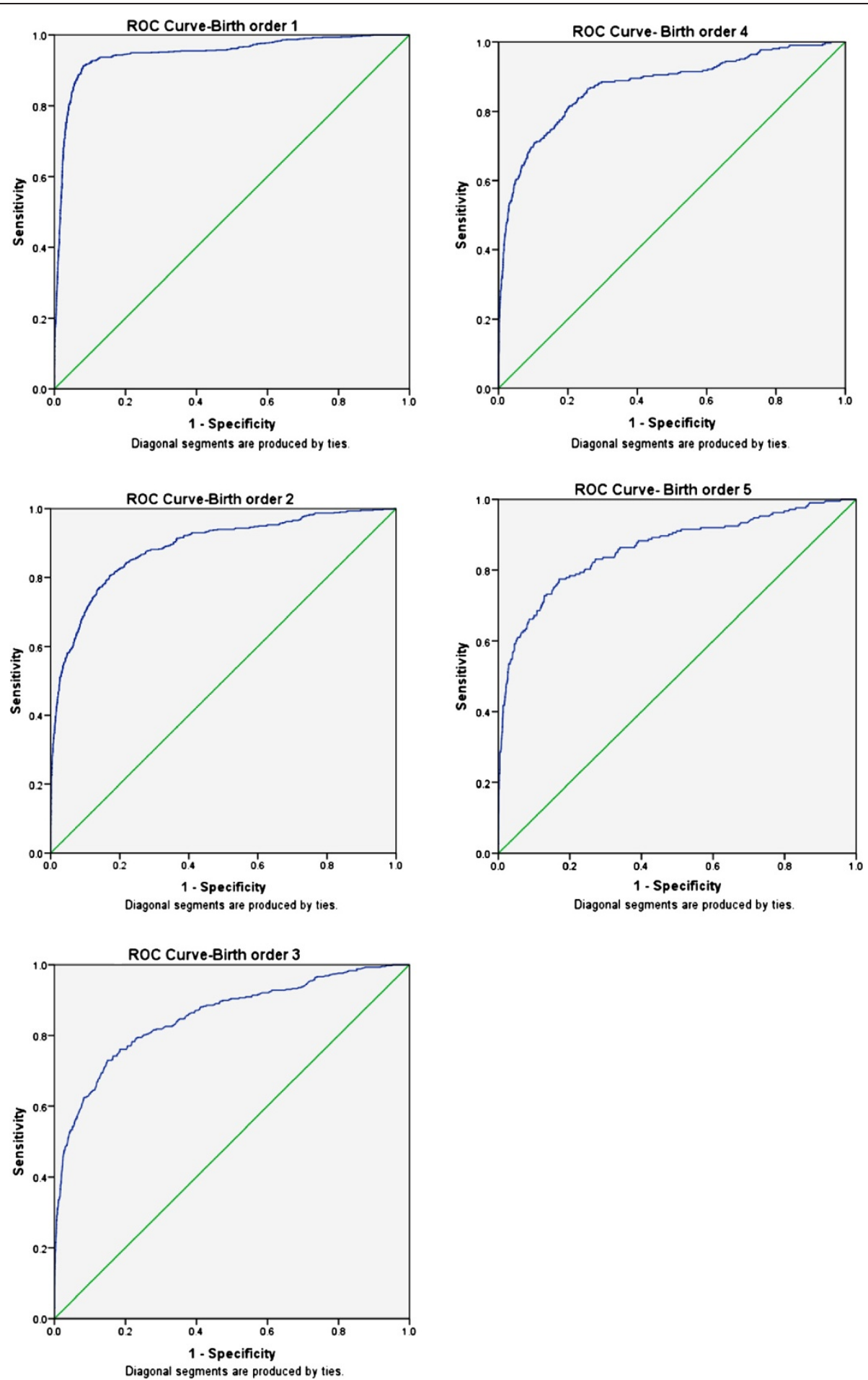

Figure 1 ROC Curves for birth order 1 to 5 .

and counsel parents at higher birth order against gender bias.

Wealth inequalities have been shown to effect underfive survival (Chalasani 2012; Mazumdar 2010; Pradhan et al. 2010; Houweling 2012). Under-five mortality is significantly higher in middle, richer and richest wealth index compared to those in poor and poorest wealth index for birth order 1 to 3 . However, it is not statistically significant in birth order 4 and 5 . It is the same with standard of living. However, wealth index is a 
protective factor in multivariate analysis for all birth orders. This is important since wealth or economic wellbeing of the family effects general health of mother and child. It is important to counsel and follow-up those in the lesser privileged sections of society in order to reduce under-five mortality.

Place of birth in rural areas produces risk for birth order 1 to 3 but is not significantly higher for birth order 4 and 5 . It strengthens the notion that under-five mortality is as high in rural as urban areas for higher birth orders. It shows the need for improving counselling in urban centres and facilities in rural centres. Neonatals are more at risk of dying than children who survive greater than 1 month and greater than 1 year. Other studies also link higher mortality risk among neonatals with early childbearing trends and lower utilization of maternity services (Kumar et al. 2013; Hall 2005). Our study supports that neonatals are at the highest risk in all birth orders.

Breastfeeding health nutrition education has no effect in birth order 2, 3, 4 and 5. Pregnancy health nutrition education has no effect in birth order 1 but improved under-five survival for all subsequent birth orders. However, neither factor is significant in multivariate analysis though this may be due to large number of missing observations. However, efforts to increase these educational indicators should continue to improve under-five survival.

Under-five survival is better in hospitals than home deliveries for birth order 1 to 3 but is not statistically significant in birth order 4 and 5 . However, there is proportional rise in mortality in hospitals and other facilities for birth order 4 and 5, maybe due to complications or due to delay in getting to the hospital. Under-five survival is significantly better in birth order 1 for antenatal care in private hospitals compared to home deliveries. However, the factor was not statistically significant in any other birth order. It has been noted that threequarters of births in rural India continue to take place at home. Mishra and Retherford have also noted that institutional antenatal care and assistance at delivery have a large effect on under-five mortality (Mishra et al. 2008). Information regarding complications in pregnancy is a protective factor in birth order 1 with under-five survival being better in those who suffer a complication. This is also borne out in multivariate analysis since information regarding complications in pregnancy leads to antenatal interventions and better care. Prenatal care is a significant factor in under-five survival for birth order 1 and 2 . It shows improvement in birth order 3 and 4 but is not statistically significant. It is a significant variable for birth order 1 in multivariate analysis. This is an important finding since it indicates that prenatal care makes a difference in under-five survival. This is in conjunction with established literature that states the dangers are higher for nulliparous compared to higher parities (Gubhaju 1985; Nankabirwa et al. 2011).

Apart from these maternal factors, our study also indicates the importance of father's education in under-five survival. The higher the father's education, the better the chances of survival for the child from birth order 1 to 5 . It is statistically significant that under-five survival is better in secondary and higher educated fathers for birth order 1 to 3 compared to no formal education and primary educated. This is a pointer to the need to educate fathers who are illiterate and/ or studied up to primary level in matters of reproductive and child health. This is supported by a study by Boone and Khan (Boone et al. 2006). However, it is not a significant factor in multivariate analysis. Father's occupation is not statistically significant in any birth order since it does not directly affect maternal or child health.

The AUROC is used to see if the model fits the data. Prediction models demonstrated high discrimination with a value of 0.91 indicating models fit the data. This demonstrates that the developed models are able to reliably predict the association between exposure variables and maternal factors contributing to under-five mortality extracted from NFHS-3 survey data.

\section{Strengths}

The study captures $92 \%$ of the total births during 59 months preceding the survey for the analysis. Thematically, the study is new in segregating information according to birth order ( 1 to 5 ) to review the determinants of under-five mortality with a view to improve maternal health care services in India. The predictive accuracy of developed models was above $80 \%$ for all developed models indicating a high accuracy to predict the outcome.

\section{Limitations}

The data is based upon recall by the mother and is open to recall biases and undocumented social pressures that lead them to say the "right" thing. The effects of exclusive breastfeeding and total versus partial immunization could not be commented upon due to the restrictions of including breastfeeding and immunization as yes and no dichotomous variables in the analysis. Nutritional differences could also be responsible for differences in underfive survival in religious groups, Christians and Muslims faring better than Hindus. However, our study did not establish a direct link between nutrition and religion though it noted that pregnancy health nutrition education acted as a protective factor. Although contribution of the explanatory variables are statistically significant, predicting mortality accounted for a Nagelkereke $R^{2}$ of 0.56, 0.37, 0.33, 0.34 and 0.34 for the developed models 1 to 5 , respectively, indicating small effect. 


\section{Conclusion and programme implications}

The study reveals the determinants of under-five mortality according to birth order. While some factors such as breastfeeding and birth interval greater than 24 months are important in under-five survival, several others such as mother's education and rural- urban place of residence show weaker relationship at higher birth orders. Efforts are needed to educate young women in matters of reproductive health and the benefits of delaying pregnancies. Maternal education, both in formal and informal sectors, can improve their utilization of antenatal care services. Information regarding complications in pregnancy acts as a protective factor for this reason. Pregnancy Health Nutrition education improves underfive survival for birth orders 2 to 5 . It is felt that prenatal care and general anxiousness related to first pregnancy that leads couples to seek early care is important in under-five survival. There is need to educate couples regarding correct nutrition, benefits of breastfeeding and immunization and possible complications so that the couples can decide and take precautions. However, complacency at higher birth orders leads to higher rates of under-five mortality. Higher Wealth index is an important indicator in under-five survival. Most parents are not specific about child's gender in the first birth order but more girl children die at higher birth orders. This clearly points to how gender preferences play a role in under-five mortality. The study identifies various focus groups for increased counselling such as mothers working in the agricultural sector and severely anaemic mothers. However, the study also shows that under-five mortality is as high in rural as urban areas for higher birth orders. It points to the need of IEC (Information, Education and Communication) network to be further strengthened to reduce under-five mortality across the board for higher birth orders.

\section{Ethical approval}

No identifying information is available in the data precluding the need for any ethical approvals. We wish to thank the DHS for making this data available for our study.

\section{Endnote}

${ }^{a}$ Birth order (or parity) refers to the numerical order of the live birth or foetal death, recorded in relation to all previous issue of the mother, irrespective of whether the issue is a live birth or foetal death or whether pregnancies were nuptial or extra-nuptial.

\section{Competing interests}

There are no declared competing interests by either of the authors.

\section{Authors' contributions}

Both authors equally contributed to the conceptualization, analysis, interpretation and writing of the paper. Both authors read and approved the final manuscript.

\section{Author details}

${ }^{1}$ Cardiology Research Centre, Heart Hospital, Hamad Medical Corporation (HMC), Doha, Qatar. '2Department of Mathematics \& Statistics, The Faculty of Science and Technology, The University of the West Indies, St Augustine Campus, Trinidad \& Tobago, West Indies.

Received: 16 April 2013 Accepted: 21 June 2013

Published: 27 June 2013

\section{References}

Arokiasamy P (2004) Regional patterns of sex bias and excess female child mortality in India. Population (English Edition) 59:833-863. doi:10.3917/ pope.406.0831

Bassani DG, Kumar R, Awasthi S, Morris SK, Paul VK, Shet A et al (2010) Causes of neonatal and child mortality in India: a nationally representative mortality survey. Lancet 376:1853-1860

Bewick V, Cheek L, Ball J (2005) Statistics review 14: logistic regression. Crit Care 9(1):112-118

Bhalotra S, Valente C, Soest AV (2009) The puzzle of Muslim advantage in child survival in India. IZA Discussion Paper No. 4009. Institute for the Study of Labor (IZA), Bonn

Bhaumik S (2013) Child mortality: will India achieve the 2015 target? BMJ 346: $f 1502$

Boone P, Zhan Z (2006) Lowering child mortality in poor countries: the power of knowledgeable parents. CEPDP, 751. Centre for Economic Performance, London School of Economics and Political Science, London, UK, ISBN 0753020572

Chalasani S (2012) Understanding wealth-based inequalities in child health in India: a decomposition approach. Soc Sci Med 75(12):2160-2169. doi:10.1016/j.socscimed.2012.08.012

Gakidou E, Cowling K, Lozano R, Murray CJL (2010) Increased education attainment and its effect on child mortality in 175 countries between 1970 and 2009: A systematic analysis. Lancet 376:959-974

Gareth J, Richard SW, Robert BE, Zulfiqar BA, Saul MS (2003) How many child deaths can we prevent this year? Lancet 2003(362):65-71

Glasier A, Gülmezoglu AM, Schmid GP, Moreno CG, Van Look PF (2006) Sexual and reproductive health: a matter of life and death. WHO, Geneva, http://www.who.int/reproductivehealth/publications/general/lancet_1.pdf. (accessed April 16 2013)

Gubhaju BB (1985) Effects of birth order and maternal age on infant and child mortality in rural Nepal. Biol Soc 2(1):15-22

Hall S (2005) Neonatal Mortality in Developing Countries: What can we learn from DHS data? Southampton Statistical Sciences Research Institute, (S3RI Applications and Policy Working Papers, (A05/02)), Southampton, UK

Headey DA, Chiu A, Kadiyala S (2012) Agriculture's Role in the Indian enigma: help or hindrance to the malnutrition crisis? Food Security 4(1):87-102

Houweling TAJ, Kunst AE (2012) Socio-economic inequalities in childhood mortality in low- and middle-income countries: a review of the international evidence. Br Med Bull 93:7-26

Jha P, Kumar R, Vasa P, Dhingra N, Thiruchelvam D, Moineddin R (2006) Low male-to-female sex ratio of children born in India: National survey of 1.1 million households. Lancet 367:211-218

Kleinbaum DG, Kupper LL, Muller KE, Nizam A (1998) Applied Regression Analysis and Multivariable Method, 3rd edn. Duxbury Press 10 Davis Drive, Belmont, CA

Kumar C, Singh PK, Rai RK, Singh L (2013) Early Neonatal Mortality in India, 1990-2006. J Community Health 38(1):120-130

Mazumdar S (2010) Determinants of inequality in child malnutrition in India. Asian Popul Stud 6:307-333

Mishra V, Retherford RD (2008) The effect of antenatal care on professional assistance at delivery in rural India. Popul Res Policy Pev 27:307-320

Mustafa HE, Odimegwu C (2008) Socioeconomic determinants of infant mortality in Kenya: analysis of Kenya DHS 2003. J Hum Soc Sc 2(2):1-16

Nankabirwa V, Tumwine JK, Tylleskär T, Nankunda J, Sommerfelt H et al (2011) Perinatal mortality in eastern Uganda: a community based prospective cohort study. PLoS One 6(5):e19674. doi:10.1371/journal.pone.0019674

National Family Health Survey (NFHS-3), 2005-06: India. Vol. I and II (2007) Mumbai. International Institute for Population Sciences, India

Pradhan J, Arokiasamy P (2010) Socio-economic inequalities in child survival in India: a decomposition analysis. Health Policy 98:114-120 
Robert BE, Saul MS, Jennifer B (2003) Where and why are 10 million children dying every year? Lancet 361:2226-2234

Sawyer CC (2012) Child mortality estimation: estimating sex differences in childhood mortality since the 1970s. PLoS Med 9(8):e1001287. doi:10.1371/ journal.pmed.1001287

Singh R, Tripathi V, Singh K, Ahuja RK, Kalai-Vani M, Dwivedi SN (2012a) Breastfeeding as a time-varying-time-dependent factor for birth spacing: multivariate models with validations and predictions. World Health Popul 13(3):28-52, 10.12927.whp.2012.22857

Singh R, Tripathi V, Kalai-Vani M, Singh K, Dwivedi SN (2012b) Determinants of birth intervals in Tamil Nadu in India: developing Cox hazard models with validations and predictions. Revista Colombiana de Estadística 35(2):289-307 (2011) SPSS 20 version. SPSS Inc. 223 South Waker Drive, Chicago IL, US

Srinivasan K (1980) Birth interval analysis in fertility surveys. WFS Scientific reports, Number, 7

United Nations Children's Fund (UNICEF) (2008) The state of Asia-Pacific's children 2008: Child survival. United Nations Children's Fund, Bangkok, Thailand

United Nations Children's Fund (UNICEF) (2012) Committing to child survival: A Promise Renewed - Progress report 2012. United Nations Children's Fund New York, NY

WHO Collaborative Study Team on the Role of Breastfeeding on the Prevention of Infant Mortality (2000) Effect of breastfeeding on infant and child mortality due to infectious diseases in less developed countries: a pooled analysis. Lancet 355:451-455

doi:10.1186/2193-1801-2-284

Cite this article as: Singh and Tripathi: Maternal factors contributing to under-five mortality at birth order 1 to 5 in India: a comprehensive multivariate study. SpringerPlus 2013 2:284.

\section{Submit your manuscript to a SpringerOpen ${ }^{\circ}$ journal and benefit from:}

- Convenient online submission

- Rigorous peer review

- Immediate publication on acceptance

- Open access: articles freely available online

- High visibility within the field

- Retaining the copyright to your article

Submit your next manuscript at $\gg$ springeropen.com 